\title{
Ações para retomada da periodicidade na publicação de revista científica
}

\author{
Rodrigo César Santiago \\ Hospital Universitário da Universidade Federal de Juiz de Fora \\ ortorodrigosantiago@gmail.com \\ Daniel Amaral Alves Marlière \\ Hospital Universitário da Universidade Federal de Juiz de Fora \\ ctbmf.marliere@gmail.com \\ Graciela Paula do Nascimento Duque \\ Hospital Universitário da Universidade Federal de Juiz de Fora \\ graciela.duque@yahoo.com.br \\ Hospital Universitário da Universidade Federal de Juiz de Fora \\ Angela Maria Gollner \\ Hospital Universitário da Universidade Federal de Juiz de Fora \\ angela.gollner@gmail.com \\ Hélady Sanders-Pinheiro \\ Hospital Universitário da Universidade Federal de Juiz de Fora \\ heladysanders@gmail.com
}

\section{Como citar:}

SANTIAGO, R. C. et al. Ações para retomada da periodicidade na publicação de revista científica. In: ABEC MEETING, 2, 2018, São Paulo. Anais... São Paulo: Associação Brasileira de Editores Científicos, 2018. p. 1-6.

http://dx.doi.org/10.21452/abecmeeting.2018.178

\section{RESUMO}

O presente estudo teve como objetivo descrever as ações utilizadas para a retomada da periodicidade das publicações de Revista Científica da área de Saúde, gerenciada pelo sistema OJS, criada em 1974. Material e métodos: Foi criado Grupo de Trabalho (GT), composto por sete integrantes, para atuar no diagnóstico do problema, mapeamento das causas potenciais, estabelecimento das estratégias para retomada do Fluxo Editorial e melhor compreensão das etapas envolvidas no processo de submissão. O GT realizou reuniões semanais, com análise de todos os artigos identificados como ativos no sistema, além de cursos de capacitação para domínio do software OJS utilizado para a editoração do periódico. Resultados: No início das atividades do GT, havia 69 artigos sem designação, 49 em avaliação e 08 em edição. Vários Editores de Seção não estavam ativos no periódico e a maior parte dos avaliadores não realizavam suas atividades dentro do prazo pré-estabelecido. Observamos a necessidade de desenvolvimento de normas e instruções mais claras e objetivas aos autores, além de tutorial para revisores e editores. Todos os resumos dos artigos não designados foram analisados pelo GT e aqueles que não atingiam a mínima qualidade metodológica foram rejeitados. Foram designados novos Avaliadores e Editores de Seção, e com os últimos realizadas reuniões regulares para agilização dos problemas encontrados. O GT se reúne regularmente uma vez por semana. Após 4 meses de atuação, a Revista apresentava no sistema 03 artigos não designados, 48 em avaliação e 13 em edição. $O$ integrantes do 
Grupo de Trabalho concluíram o Curso OJS versão 3, o que permitiu uma melhoria do fluxo e processo editoriais. Conclusão: O GT desenhou um diagnóstico da situação do periódico e dos pontos a serem alvo da ação. Para um fluxo editorial eficiente, há a necessidade de uma adequada comunicação entre todos os envolvidos no processo e um comprometimento por parte dos mesmos, visto que em sua grande maioria, os trabalhos de editoração e publicação em periódicos científicos é exercido de maneira voluntária e envolve conhecimento e comprometimento científico.

Palavras chaves: Fluxo editorial. Revista científica. Metodologia.

\section{ABSTRACT}

The aim of the present study was to describe the actions used for resuming publication periodicity of a Scientific Journal from Health area created in 1974, under the Open Journal System (OJS) management. Material and Methods: A work group (WG) was created with 07 participants to define the problem's diagnosis, look for the causes, establish the strategies for resuming editorial flow and better comprehension about the steps involved during the submission procedures. The WG performed meeting weekly for analyzing every manuscript identified as active on the system. In addition, the WG had been training in OJS courses. Results: There were 69 articles as "not designated", 49 "under evaluation" and 08 for text edition. Many Editors of Section were not acting and most of the reviewers did not do their own revisions in the pre-set time. The WG had observed needs new rules and instructions for authors, reviewers, and editors. Thus, the WG had to develop guidelines for authors and tutorials for reviewers and editors. All "not designated" articles were evaluated by reading every abstract in which any were rejected because did not reach a minimal methodological quality. New reviewers and section editors were designated to solve specific problems by meetings with WG once a week. After 4 months, there were only 03 articles as "not designated", 48 under revision and 13 for edition at the Journal's system. The WG members concluded the OJS course (version 3.0). Conclusions: The WG found out a diagnosis about journal situation and established the actions to solve the problems. For an efficient editorial flow, there are needs for adequate communication between all involved in the process, and editors and reviewers must be more commitment, hence most of the publishing workers in scientific journals are carried out on a voluntary basis and involves scientific commitment and knowledge.

Keywords: Editorial flow. Cientific journal. Methodology.

\section{INTRODUÇÃO}

A introdução das Tecnologias de Informação e Comunicação (TICs) trouxe mudanças nos meios eletrônicos e influenciou o comportamento do usuário da informação: não somente o suporte da informação mudou, mas também a maneira pela qual o conhecimento é disseminado e tratado. Os periódicos científicos são um exemplo que ilustra adequadamente essa mudança. Cada vez mais títulos de revistas científicas são disponibilizados no meio eletrônico (FERREIRA; CARREGNATO, 2008).

Uma das características desejadas do periódico eletrônico é a maior rapidez em disponibilizar os artigos para o público, tendo em vista que o processo de impressão e distribuição é inexistente na publicação on-line. Todavia, algumas etapas editoriais, como a avaliação por pares, por exemplo, continuam consumindo grande tempo do processo editorial. Apesar da comunicação instantânea por meio da transmissão eletrônica das mensagens, os atrasos de tempo se devem em grande parte à intervenção humana nos processos de revisão e edição, dentre outros (KING; TENOPIR, 1998).

Tendo em vista que a demora no processo de editoração comprometem o fluxo de publicação dos artigos e a perda de periodicidade, além da indexação do periódico nas bases de dados relevantes, o presente estudo teve como objetivo descrever metodologia desenvolvida para a retomada da continuidade da publicação científica de periódico nacional, bem como reestabelecer fluxo editorial eficiente, com reestruturação do corpo de editores e avaliadores e definições de diretrizes para 
guiar tanto os autores como os revisores durante as submissões.

\section{MATERIAL E MÉTODOS}

O periódico envolvido no presente estudo foi a HU Revista do Hospital Universitário (HU) da Universidade Federal de Juiz de Fora (UFJF) em Minas Gerais (MG), e do Programa de Pós-Graduação em Saúde (PPgS) da mesma. A Revista foi criada em 1974, tendo como escopo de abrangência as áreas da Saúde. Migrou para os sistema Open Journal Systems (OJS) em 1996 e teve três editores-chefes desde sua criação.

A partir de fevereiro de 2018 foram disponibilizados novos colaboradores contratados para atuar na Empresa Brasileira de Serviços Hospitalares (EBSERH) criando assim condições para a reestruturação do periódico. Foi então criado Grupo de Trabalho (GT) para a HU Revista com este objetivo, em 08 de fevereiro de 2018, composto por 08 membros, sendo: 02 professores Doutores da UFJF, 05 empregados da EBSERH (01 Doutor, 03 Mestres e 01 Graduado) e um Bolsista de graduação (UFJF). O grupo foi nomeado pelo superintendente do $\mathrm{HU}$ com autonomia para tomada de decisões.

Serão descritas as ações realizadas e os resultados obtidos no período de atuação entre feverreiro de 2018 a junho de 2018.

\section{RESULTADOS E DISCUSSÃO}

Dos oito integrantes nomeados para o GT, cinco atuaram efetivamente no período descrito neste estudo. $O$ grupo passou a se reunir semanalmente para linearização e atualização das ações. Foi desenvolvido, nos primeiros 3 meses, relatório com o diagnóstico da situação do periódico e um plano de trabalho com o planejamento de atividades para período de 18 meses.

Artigos submetidos: Foram quantificados os artigos encontrados no sistema, em cada uma das 03 categorias: "sem designação de editor", "em avaliação" e "em edição". No início das atividades do GT havia 65 artigos "sem designação", 49 "em avaliação" e 09 artigos em "processo de edição".

Foi feita leitura pelo GT dos resumos e metodologia dos artigos sem designação (65), para análise básica de adequação de metodologia científica. Os que preenchiam requisitos básicos eram encaminhados para avaliação por pares. Foram rejeitados dois terços dos artigos. Dos artigos em avaliação, foi analisado o estágio atual da revisão e tomadas medidas necessárias para o prosseguimento do processo de submissão. Em relação aos artigos em edição, foram retomados os processos de edição de texto e layout.

Os artigos novos submetidos no sistema, a partir de março de 2018, passaram a ser avaliados inicialmente pelo GT, que, posteriormente, decidia, baseado na adequação de metodologia científica, entre (1) rejeitar; (2) devolver para formatação inicial ou (3) encaminhar para avaliação. Essa avaliação inicial era realizada em reuniões semanais do GT.

Editores de seção: Foi solicitado, por e-mail, posicionamento em relação à continuidade da atividade de editoração na revista. Dos 10 editores ativos, apenas cinco manifestaram interesse em continuar. Verificamos, pela análise dos artigos em avaliação que a velocidade de solicitação de revisão por pares, comunicação 
entre autores e editores era lenta e não padronizada. Alguns e-mails padrão não eram claros, assim como a informação sobre as normas de formatação de artigo. Em seguida, foram executadas reuniões com os Editores de Seção que possuíam artigos em avaliação sob sua responsabilidade. Além disso, nove novos Editores de Seção foram convidados para participar da revista e com estes realizamos reuniões regulares para familiarização do uso do sistema OJS.

Avaliadores: Havia 642 avaliadores cadastrados no sistema da HU Revista. Observou-se que alguns não acessavam o sistema ou não exerciam a atividade há anos. Todos os avaliadores foram notificados, via e-mail, com orientações para efetuarem seu recadastramento e terem sua função mantida no sistema. Somente $18 \%$ desses responderam ao e-mail e um percentual ainda menor realizou as alterações solicitadas em seu perfil, com o preenchimento de campos obrigatórios como ORCID e URL do Currículo Lattes.

Capacitação da equipe: Curso sobre o sistema OJS, software utilizado no processo de editoração da HU Revista, na versão 2.4 .3 foi realizado por três integrantes do GT. Várias falhas e limitações foram identificadas na configuração online da HU Revista. A mesma equipe deu início ao novo curso, agora na versão OJS 3.0. Existem várias alterações necessárias que serão implementadas na Revista após a conclusão do mesmo, tais como novo guia para autores e avaliadores, novas fichas para avaliação dos artigos, novos modelos de e-mail para comunicação entre Editor/Autor/Avaliador, além de modificações na própria pagina da Revista on-line, a começar pela unificação do acesso.

O Fluxo editorial atual encontra-se ilustrado na Figura 1. Após 04 meses de trabalho, a situação atual do sistema (30/06/2018) mostra 03 artigos "sem designação", 46 "em avaliação" e 14 artigos em processo de edição. Aparentemente, não houve redução considerável do número de artigos "em avaliação", o que poderia levantar dúvidas sobre a eficácia do modelo adotado. Entretanto, o que se observou foi que nos quatro meses de atividade do GT, 29 novos artigos entraram no sistema como "não designados". Para a categoria "em avaliação", 26 novos artigos que já estavam no sistema ou entraram após início das atividades do Grupo de Trabalho, foram designados para esta categoria. Desta forma, houve redução considerável do número de artigos "em avaliação", sendo 05 deles encaminhados para edição e 24 excluídos após revisão por pares (Figura 2). 
Figura 1- Fluxo Editorial adotado pelo Grupo de Trabalho.

\section{FLUXO EDITORIAL - HU Revista}

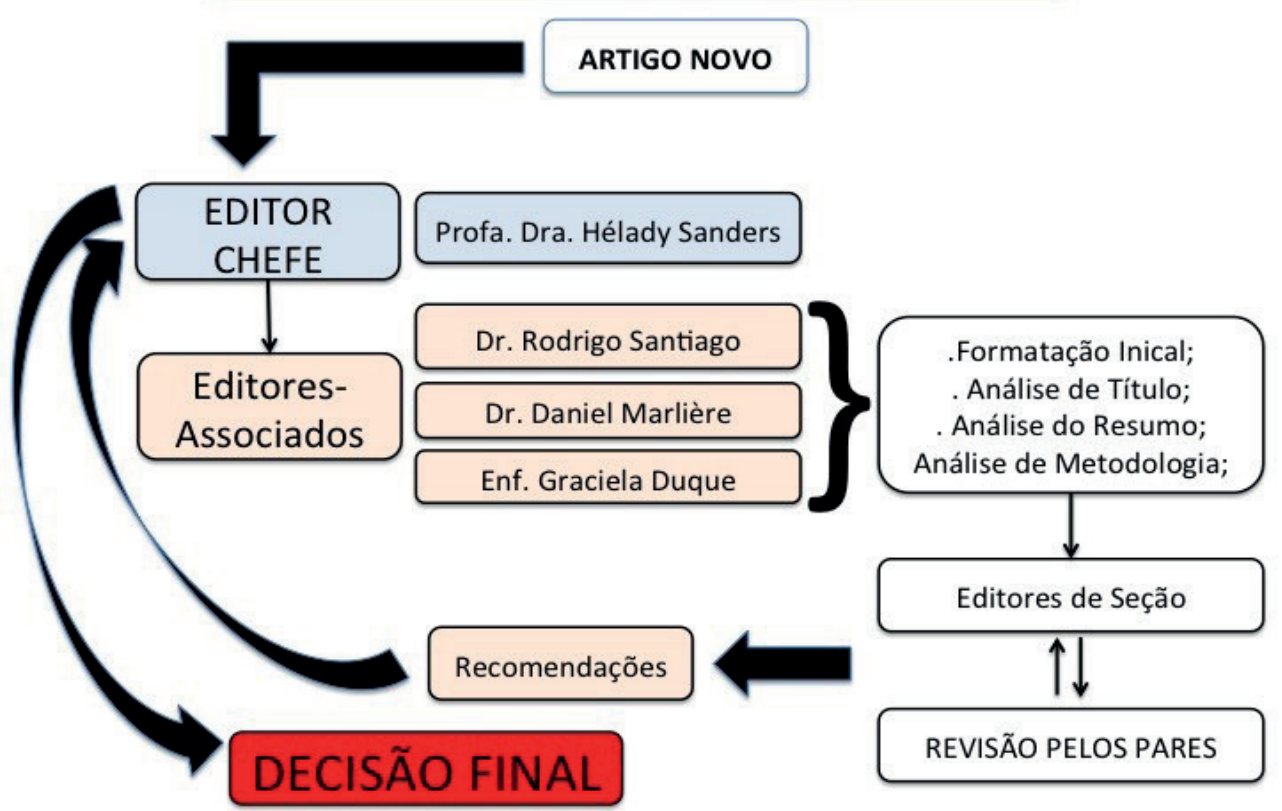

Elaborado pelos autores, 2018.

Há problemas a serem abordados e ações a serem implementadas em médio prazo, cujo conteúdo está organizado no plano de trabalho. Dentre elas destacamos a atualização do regimento, atualização dos dados da revista nas bases de indexação, migração para versão atualizada do sistema OJS 3, revisão do conteúdo do site,e adoção do DOI.

Figura 2 - Fluxo dos artigos no sistema durante ação do GT.

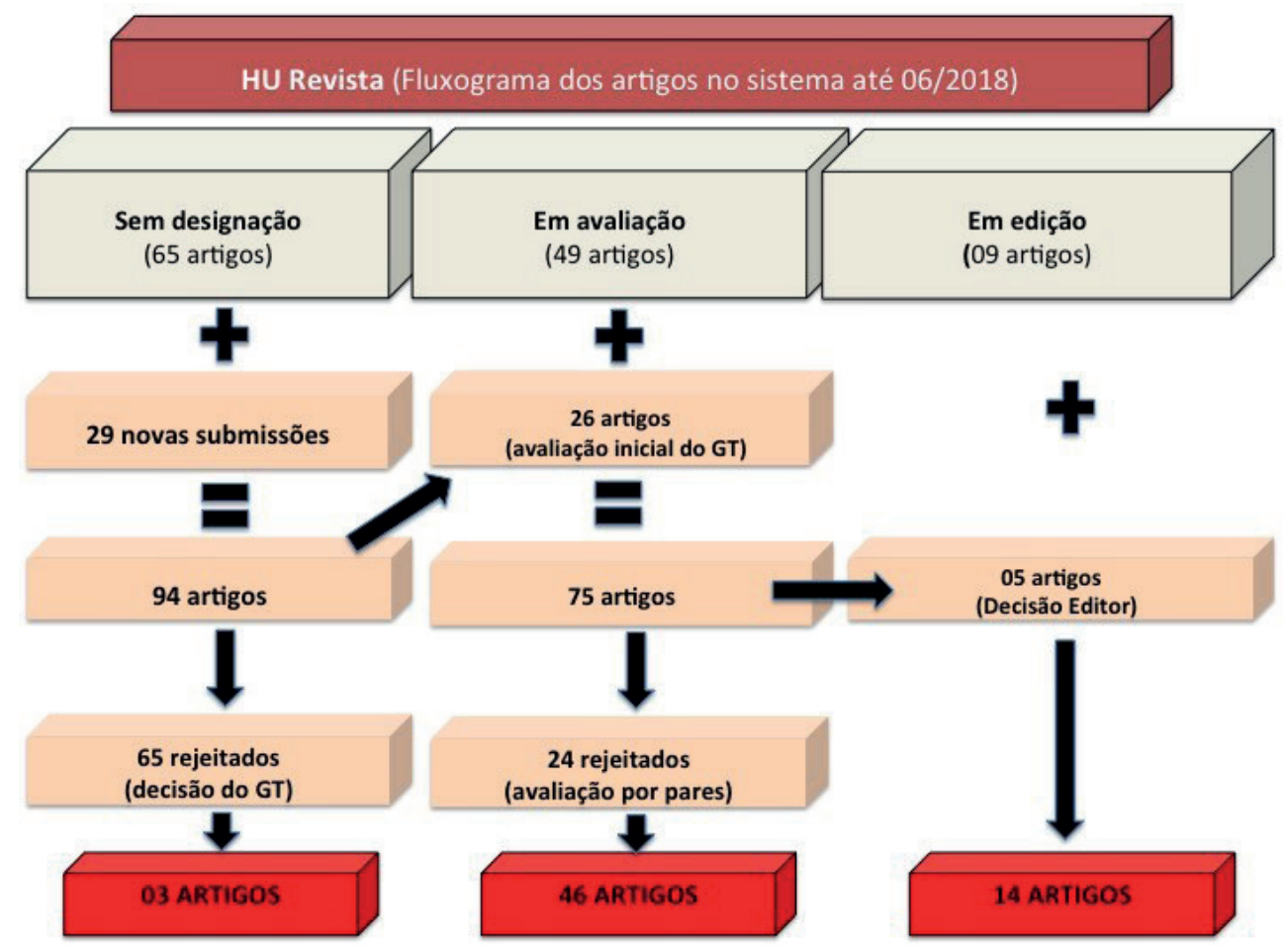

Elaborado pelos autores, 2018. 


\section{CONCLUSÃO}

O processo de Fluxo Editorial é complexo e exige grande esforço por parte de todos envolvidos. O modelo adotado no presente estudo mostrou-se eficaz ao observarmos a evolução do trabalho acumulado no sistema. O completo entendimento sobre o sistema de editoração OJS permitirá aprimorar todo o processo de submissão, customização do acesso on-line da HU Revista, além de uma redução do tempo despendido entre a entrada do manuscrito no sistema até a decisão editorial final.

\section{REFERÊNCIAS}

FERREIRA, A. G. C.; CAREGNATO, S. E. A editoração eletrônica de revistas científicas brasileiras: o uso de SEER/OJS. TransInformação, v. 20, n. 2, p. 171180, 2008. http://dx.doi.org/10.1590/S0103-37862008000200005.

KING, D. W.; TENOPIR, C. A publicação de revistas eletrônicas: economia da produção, distribuição e uso. Ciência da informação, v. 27, n. 2, 1998. Disponível em: <http://www.brapci.inf.br/v/a/713 >. Acesso em: 30 abr. 2018. 